\title{
Metabolic syndrome as a risk factor for coronary heart disease and stroke: An 11-year prospective cohort in Taiwan community
}

\author{
Kuo-Liong Chien ${ }^{\mathrm{a}, \mathrm{b}}$, Hsiu-Ching Hsu ${ }^{\mathrm{b}}$, Fung-Chang Sung ${ }^{\mathrm{c}}$, \\ Ta-Chen Su ${ }^{\mathrm{b}}$, Ming-Fong Chen ${ }^{\mathrm{b}}$, Yuan-Teh Lee ${ }^{\mathrm{b}, \mathrm{d}, *}$ \\ ${ }^{a}$ Institute of Preventive Medicine, School of Public Health, National Taiwan University, Taiwan \\ ${ }^{\mathrm{b}}$ Department of Internal Medicine, National Taiwan University Hospital, Taipei, Taiwan \\ ${ }^{c}$ Institute of Environmental Medicine, College of Public Health, National Taiwan University, Taipei, Taiwan \\ d Ming-Shen Medical Center, Taoyuang, Taiwan
}

Received 25 December 2005; received in revised form 15 June 2006; accepted 26 July 2006

Available online 15 September 2006

\begin{abstract}
Objective: Patterns of metabolic syndrome among populations in the Asia-Pacific region remain unknown. This study sought to establish the prevalence of metabolic syndrome in Taiwan and the risk of cardiovascular disease imparted by this syndrome.

Methods and results: A cohort of 3602 individuals aged 35 years and above from one Taiwan community in 1990-1991 was followed up for a median of 9.0 years (inter-quartile range: 7.9-10.0 years). Women had a higher prevalence rates of age-standardized metabolic syndrome than men $(28.9 \%$ versus $16.6 \%)$ by original NCEP-ATP III criteria, and the rates increased progressively. As numbers of metabolic syndrome increased, the hazard risk (HR) increased significantly, up to 5.5 (95\% confidence interval [CI], 2.2-13.7) for coronary heart disease and 3.5 (95\% CI, 1.9-6.5) for stroke. We found that low HDL cholesterol and high BMI had the highest risk for coronary heart disease (HR, 2.3), followed by metabolic syndrome (HR, 1.8). For stroke events, high blood pressure had the highest risk (HR, 3.0), also followed by metabolic syndrome (HR, 2.1). The highest attributable risk for the general population was low HDL cholesterol for coronary heart disease event and high blood pressure for stroke.

Conclusions: Metabolic syndrome was highly prevalent among the adult population and associated with an increased risk for coronary heart disease and stroke.
\end{abstract}

(C) 2006 Elsevier Ireland Ltd. All rights reserved.

Keywords: Metabolic syndrome; Coronary heart disease; Stroke; Community study

\section{Introduction}

Coronary heart disease (CHD) and stroke constitute a global public health burden. Prevention strategy should be implemented during the early atherosclerosis phase. Insulin resistance impairs tissue energy utilization and elicits metabolic derangement during the early phase of atherosclerosis [1,2]. Metabolic syndrome clusters together and had strong enhancement with the risk of diabetes [3,4]. Incidences of diabetes increased progressively globally, espe-

\footnotetext{
* Corresponding author. Tel.: +886 22356 2830; fax: +886 223920456.

E-mail address: ytlee@ha.mc.ntu.edu.tw (Y.-T. Lee).
}

cially in Asia-Pacific countries [5]. The Third Report of the National Cholesterol Education Program's Adult Treatment Panel has proposed a general working criterion for metabolic syndrome, eliciting important risk factors for monitoring community-based intervention programs [6].

The incidences of CHD were lower and those of stroke were higher in the Asia-Pacific countries than in Western countries. Asian-Pacific countries use the lower body mass index as an obesity index than Caucasian people [7]. In general, the concentration of high-density lipoprotein (HDL) cholesterol is lower for Asian populations than for Caucasians, especially for men. Furthermore, by the process of urbanization and globalization, unhealthy conditions, such 
as obesity, sedentary lifestyle, and smoking, have become prevalent among Asians in recent years [8]. There has been no prospective cohort study that has investigated trends of metabolic syndrome patterns associating with CHD and stroke for the Chinese, especially in the community-based population. We investigated the prevalence rates of metabolic syndrome in a community cohort, examined the atherosclerotic risk factors serially during the follow-up period, and compared the patterns as time progressed.

\section{Materials and methods}

\subsection{Study design and population}

The Chin-Shan Community Cardiovascular Cohort study has begun in 1990, following 1703 men and 1899 women aged 35 years old and above, homogenous in Chinese ethnicity, in Northern Taiwan for the study of cardiovascular diseases $[9,10]$. The cohort was assembled from the registry data of the bureaucracy and the participants were recruited by house-to-house visits. The study was approved by the IRB in the National Taiwan University Hospital.

Participants received baseline health examination at the community health center [10]. We collected the data from the participants at baseline (1990-1991), the first followup (1992-1993), second (1994-1995), and fifth follow-up periods (1997-1998). We excluded persons with a baseline history of cardiovascular disease and missing metabolic syndrome status. A total of 3515 subjects were included in this study. We recruited the subjects by volunteer basis and respondent rates were up to $83 \%$ [10].

In the survey, all of the study participants were individually interviewed from a structured questionnaire, for the information on socio-demographic characteristics, physical activity, smoking, alcohol drinking habits, dietary characteristics, personal and family histories of diseases and hospitalizations. With informed consent, the participants underwent physical examinations and laboratory tests. The examiners undertook training in the questionnaire collections and measures. Blood pressure was taken after $10 \mathrm{~min}$ of rest and measured by a mercury sphygmomanometer with the subject seated comfortably, with the arms supported and positioned at the level of the heart. Two blood pressure readings were taken in both arms. The cuff bladder was inflated quickly and then deflated $2 \mathrm{mmHg}$ every second. If the readings were taken varied by more than $10 \mathrm{mmHg}$, an additional reading was taken. The disappearance of Korotkoff phase V sound was recorded as diastolic pressure. The average of blood pressure from both arms was used in the final analysis.

Neither smoking nor caffeine was permitted before the blood pressure measurement. Body mass index (BMI) was calculated as weight (in kilograms)/height (in square meters) in baseline and serial measurements. Waist circumference was measured by a trained examiner using a measuring tape positioned at the midway between the lowest rib and the iliac crest. The measurement was made at minimal respiration status, with the tape snug but not compressing the skin.

\subsection{Blood sampling and analytic methods}

The procedures of blood sampling and analytic methods have been described previously [10]. In brief, fasting serum samples were stored at $-70^{\circ} \mathrm{C}$ prior to the batch assay using standard enzymatic tests for cholesterol and triglyceride (Merck 14354 and 14366, Germany) and precipitation methods for HDL and LDL cholesterol (Merck 14993 and 14992). Glucose levels were measured by enzymatic assay (Merck 3389) using an Eppendorf 5060 autoanalyzer.

\subsection{Definition of metabolic syndrome}

We analyzed the distribution frequency rates of various recent metabolic syndrome criteria, including original NCEP-ATPIII [6], modified Asian ATPIII [11,12], Asian IDF [13], original AHA/NHLBI [14], modified Asian AHA/NHLBI, modified BMI ATPIII and modified BMI AHA/NHLBI criteria. For original NCEP-ATPIII criteria [6], a participant with three of following was defined as having metabolic syndrome: (1) blood pressure of at least $130 / 85 \mathrm{mmHg}$ or treated hypertension; (2) serum triglyceride of at least $150 \mathrm{mg} / \mathrm{dL}$; (3) HDL cholesterol of $<40 \mathrm{mg} / \mathrm{dL}$ in men and $<50 \mathrm{mg} / \mathrm{dL}$ in women; (4) fasting glucose of $110 \mathrm{mg} / \mathrm{dL}$ or more; and (5) central obesity, waist circumference greater than $102 \mathrm{~cm}$ in men and $88 \mathrm{~cm}$ in women. Modified Asian ATPIII criteria are the same as original ATPIII except waist circumference greater than $90 \mathrm{~cm}$ in men and $80 \mathrm{~cm}$ in women [11]. Asian IDF criteria were defined by central obesity and more or equal two other components [13]. Modified Asian AHA/NHLBI criteria is the same as ATPIII criteria, with Asian central obesity cutoff and fasting glucose of $100 \mathrm{mg} / \mathrm{dL}$ or more [14]. Because of no waist circumference in the original baseline (1990-1991), we used the waist circumference of the 2nd follow-up data (1994-1995) for baseline analysis $(N=2564)$.

We used the modified BMI ATPIII criteria for predicting cardiovascular events $(n=3515)$.

\subsection{Endpoint verification}

Death due to coronary heart disease was verified by medical records, office death certificates, or household visit. In defining the endpoint of coronary heart disease events, we included coronary heart disease death, new onset of myocardial infarction, coronary artery bypass graft, and percutaneous coronary re-vascularization. We defined these hard endpoints by hospitalization and death certificate documents.

Stroke was defined as death from stroke or hospitalization due to sudden neurologic deficit of vascular origin. If the outcome classification was not clear, interview with family members were conducted in each death cases to verify. The investigators reviewed the endpoints blindly, not knowing the 
subject's medical condition. We applied the objective criteria to medical record documentation.

\subsection{Statistical analysis}

The prevalence rates of metabolic syndrome status and its components were described by gender and age, while patterns by follow-up periods were plotted. The direct agestandardized prevalence rates of metabolic syndrome were also calculated, using the world population as references [15].

Participants who were cases of CHD or stroke in baseline were excluded. Kaplan-Meir curves free from coronary heart disease events by status of metabolic syndrome and numbers at were plotted and the logrank test was used to test the difference. The effects of metabolic syndrome components on cardiovascular events were determined by Cox proportional hazards model, adjusting for age, smoking, irregular physical activity and exercise habits (yes versus no, defined by less than 20 min exercise in every week) and alcohol drinking (yes versus no, defined by frequent alcohol drinking) status. The proportionality assumption and the fit of the proportional hazards model were verified graphically and tested by the Grambsch and Therneau statistics [16].

The relative importance of various metabolic syndrome components for predicting CHD and stroke events were compared by three tests. The first was to test the magnitude of regression coefficients (hazard risk comparison) for each binary risk factors, and these coefficients were tested for the equality of hazard risks [17]. The population attributable risk (PAR) fraction was used to estimate the effects attributed to the change in the risk proportion of the population.

Second, discrimination was quantified by calculating the quantity of $c$ statistic, analogous to the area under a receiver operating characteristic (ROC) curve [18]. The area-undercurve statistics showed the probability in the model and assigned a high risk to those who developed events than to those who did not. We also compared the areas under two receiver operating curves graphically by sex.

Third, the Hosmer-Lemeshow Chi-square statistic was used to test the agreement between predicted and observed outcomes based on calibration ability, dividing the cohort population risk for CHD or stroke events in the whole study period [19]. The $P$ value less than 0.05 was considered statistically significant. All statistical analyses were performed using SAS Version 9.1 (SAS Institute, Inc., Cary, NC) and STATA version 9 (Stata Corporation, College Station, Texas).

\section{Results}

Among 3515 subjects in the cohort, the raw and agestandardized prevalence rates of metabolic syndrome by various criteria were shown in Table 1. The overall prevalence rates were increasing over the follow-up periods. Women had higher prevalence rates than men among different criteria, and the prevalence rates were higher in second follow-up period than baseline period. Among different criteria, the modified Asian AHA/NHLBI had the highest rate; the second highest was modified Asian ATPIII criteria, and the lowest was the original ATPIII criteria. The BMI-specific criteria had lower rates than its waist circumference counterpart criteria. The new AHA/NHLBI criteria rates were higher than NCEP/ATPIII criteria due to lower glucose cutoff levels in new AHA/NHLBI criteria.

Table 2 shows the average baseline age and atherosclerotic risk measured by sex and metabolic syndrome status. Women with metabolic syndrome had higher average values in age, systolic blood pressure, body mass index, total and LDL cholesterol, and fasting glucose levels. Men with metabolic syndrome had highest triglyceride and lowest HDL cholesterol. These atherosclerotic risk prevalence rates among persons with metabolic syndrome were also higher for women than men, particularly in high blood

Table 1

Raw and standardized prevalence rates of metabolic syndrome by various criteria in the study population, specified by genders and examination periods

\begin{tabular}{|c|c|c|c|c|c|c|c|}
\hline Rate $(\%)$ & $\begin{array}{l}\text { Original } \\
\text { NCEP-ATPIII }\end{array}$ & $\begin{array}{l}\text { Modified Asian } \\
\text { ATPIII }\end{array}$ & Asian IDF & $\begin{array}{l}\text { Original } \\
\text { AHA/NHLBI }\end{array}$ & $\begin{array}{l}\text { Modified Asian } \\
\text { AHA/NHLBI }\end{array}$ & $\begin{array}{l}\text { Modified BMI } \\
\text { ATPIII }\end{array}$ & $\begin{array}{l}\text { Modified BMI } \\
\text { AHA/NHLBI }\end{array}$ \\
\hline \multicolumn{8}{|l|}{ Baseline (1990-91) } \\
\hline \multicolumn{8}{|l|}{ Men } \\
\hline Raw & 16.4 & 23.0 & 16.6 & 21.6 & 28.3 & 20.0 & 25.6 \\
\hline Standardized & 16.6 & 23.5 & 17.2 & 21.7 & 28.6 & 20.2 & 25.5 \\
\hline \multicolumn{8}{|l|}{ Women } \\
\hline Raw & 30.1 & 38.0 & 38.1 & 37.0 & 44.9 & 27.8 & 34.8 \\
\hline Standardized & 28.9 & 36.7 & 36.9 & 35.6 & 43.5 & 26.4 & 33.2 \\
\hline \multicolumn{8}{|c|}{ Second follow-up (1994-95) } \\
\hline \multicolumn{8}{|l|}{ Men } \\
\hline Raw & 22.7 & 28.3 & 16.6 & 30.9 & 43.9 & 26.5 & 34.6 \\
\hline Standardized & 23.7 & 29.3 & 16.9 & 31.9 & 38.1 & 27.6 & 36.0 \\
\hline \multicolumn{8}{|l|}{ Women } \\
\hline Raw & 34.9 & 44.7 & 38.8 & 43.9 & 54.1 & 33.5 & 42.9 \\
\hline Standardized & 33.6 & 43.2 & 37.5 & 42.6 & 52.7 & 32.2 & 41.7 \\
\hline
\end{tabular}


Table 2

Basic demographic and atherosclerotic risk factors of metabolic syndrome among the study population $(N=3515)$

\begin{tabular}{|c|c|c|c|c|c|c|c|c|}
\hline \multirow[t]{4}{*}{ Variables } & \multicolumn{4}{|l|}{ Men } & \multicolumn{4}{|c|}{ Women } \\
\hline & \multicolumn{2}{|c|}{$\operatorname{MetS}(-)$} & \multicolumn{2}{|c|}{ MetS (+) } & \multicolumn{2}{|c|}{ MetS (-) } & \multicolumn{2}{|c|}{ MetS (+) } \\
\hline & \multicolumn{2}{|c|}{$N=1327$} & \multicolumn{2}{|c|}{$N=332$} & \multicolumn{2}{|c|}{$N=1340$} & \multicolumn{2}{|c|}{$N=516$} \\
\hline & Mean & S.D. & Mean & S.D. & Mean & S.D. & Mean & S.D. \\
\hline Age (year) & 55.9 & 12.4 & 55.4 & 11.6 & 52.2 & 12.1 & 58.9 & 11.5 \\
\hline Systolic blood pressure (mmHg) & 120.9 & 17.6 & 137.1 & 19.5 & 121.1 & 19.3 & 141.4 & 20.9 \\
\hline Diastolic blood pressure $(\mathrm{mmHg})$ & 75.1 & 10.3 & 84.6 & 11.0 & 74.9 & 10.3 & 83.2 & 11.0 \\
\hline Body mass index $\left(\mathrm{kg} / \mathrm{m}^{2}\right)$ & 22.2 & 2.7 & 26.0 & 3.5 & 23.0 & 3.0 & 26.3 & 3.7 \\
\hline Waist circumference $(\mathrm{cm})$ & 81.6 & 9.5 & 91.5 & 9.6 & 80.3 & 9.5 & 88.7 & 9.5 \\
\hline Total Cholesterol (mg/dL) & 188.4 & 42.1 & 209.5 & 50.8 & 197.5 & 43.7 & 217.7 & 47.2 \\
\hline Triglyceride (mg/dL) & 103.2 & 63.8 & 233.0 & 141.4 & 94.0 & 47.6 & 201.5 & 128.3 \\
\hline HDL cholesterol (mg/dL) & 48.8 & 12.6 & 36.2 & 8.7 & 52.0 & 12.0 & 39.3 & 7.5 \\
\hline LDL cholesterol (mg/dL) & 126.9 & 40.2 & 157.0 & 47.4 & 134.3 & 41.7 & 163.7 & 44.7 \\
\hline \multirow[t]{2}{*}{ Fasting glucose (mg/dL) } & 105.4 & 22.8 & 129.3 & 39.6 & 102.4 & 21.8 & 130.6 & 48.8 \\
\hline & $\mathrm{N}$ & $(\%)$ & $\mathrm{N}$ & $(\%)$ & $\mathrm{N}$ & $(\%)$ & $\mathrm{N}$ & $(\%)$ \\
\hline Smoking (Yes) & 939 & 70.8 & 239 & 72.0 & 63 & 4.7 & 35 & 6.8 \\
\hline Drinking (Yes) & 697 & 52.5 & 183 & 55.1 & 127 & 9.5 & 44 & 8.5 \\
\hline Irregular exercise habits (Yes) & 207 & 15.6 & 60 & 18.1 & 166 & 12.4 & 93 & 18.0 \\
\hline
\end{tabular}

Abbreviation: S.D., standard deviation; MetS, metabolic syndrome by modified BMI ATPIII criteria.

pressure (82\% versus 76\%) and low HDL (95\% versus $76 \%$ ). The prevalence rates of dyslipidemia and diabetes were likewise higher in metabolic syndrome. Although men had a much higher smoking rate than women, there was no significant association between smoking and metabolic syndrome.

A total of 3515 subjects, excluding baseline cardiovascular disease history and missing metabolic syndrome components, were followed up for a median of 9.0 (inter-quartile range: 7.9-10.0) years. The incidence rates of coronary heart disease events were 3.94 and 1.86 (per 1000 person years) and of stroke events were 6.24 and 4.18 (per 1000 person years) for men and women, respectively. Fig. 1 shows the prevalence of metabolic syndrome components and incidence rates for CHD and stroke by metabolic syndrome numbers in each sex. Both CHD and stroke rates increased when the number of metabolic syndrome components increased, peaking among individuals with four or five components. Also, the stroke incidence rates were higher than CHD rates, and men were at higher risk than women in all categories.

The 11-year survival rates of CHD were 94.2\% among those with metabolic syndrome and $96.9 \%$ among those without (Fig. 2). As components of metabolic syndrome increased, the 11-year survival rates of CHD events decreased progressively, from $99.3 \%$ for individuals with none of these components to $92.7 \%$ for those with four to five components. A similar pattern was also observed on stroke events with the corresponding survival rates of 96.2 and $91.7 \%$, respectively. Both survival relationships appeared to follow the dose-response trend significantly $(P<0.001)$. The Kaplan-Meir estimated curve and logrank test results showed the metabolic syndrome and numbers of components were significant risk factors for coronary heart disease events (Fig. 2).
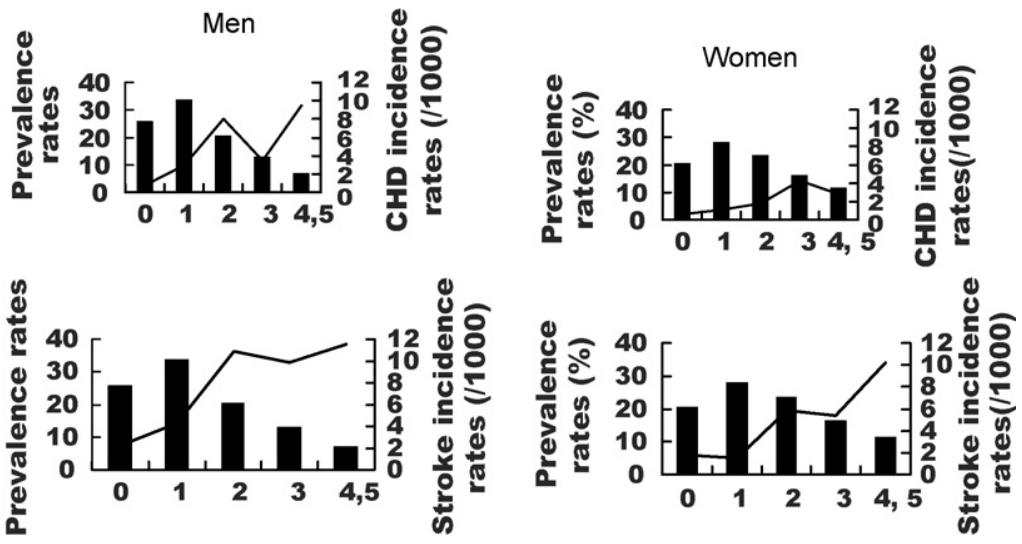

Fig. 1. Prevalence of metabolic syndrome components (bar) and incidence rates of CHD and stroke by metabolic syndrome components (line) among the study population, stratified by gender. The $X$-axis shows the number of metabolic syndrome components in baseline. 

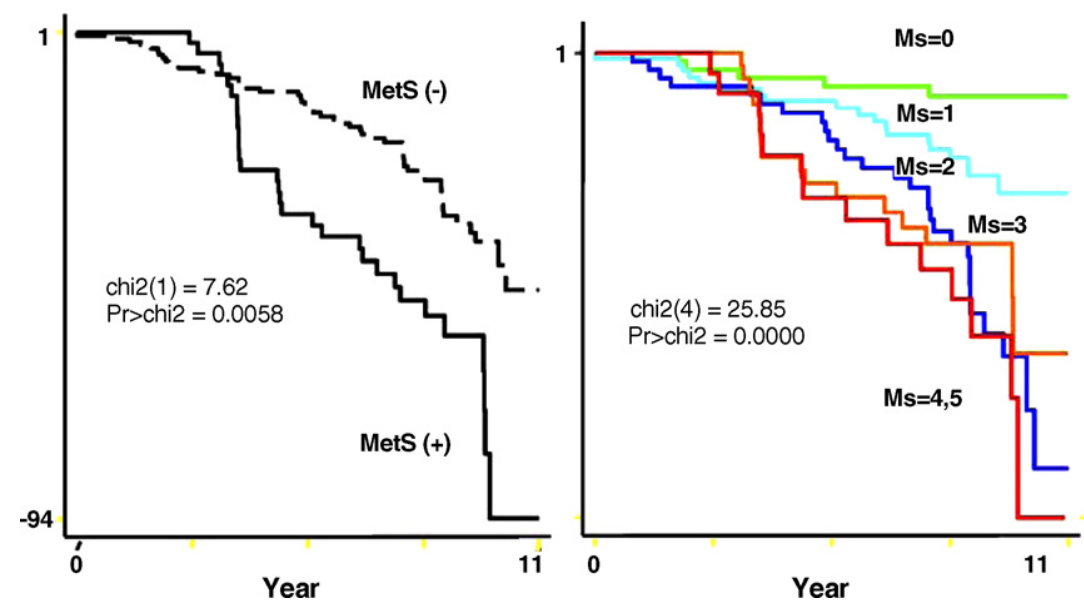

Fig. 2. Kaplan-Meir curves of 11-year free from coronary heart disease events by status of metabolic syndrome at study entry (left) and by metabolic syndrome numbers at study entry (right).

Hazard risks for CHD and stroke with the metabolic syndrome and its components, adjusted for age, gender, smoking, drinking and physical activity status, were significant in both genders (Table 3). As numbers of metabolic syndrome increased, the hazard risks (HR) increased in a significant dose-response trends to 5.5 (95\% confidence interval $[\mathrm{CI}]=2.2-13.7)$ for $\mathrm{CHD}$ and $3.5(95 \% \mathrm{CI}=1.9-6.5)$ for stroke events with four to five components. Low HDL cholesterol and high BMI were the components associated with the highest risk of CHD (HR, 2.2-2.3), followed by metabolic syndrome (HR, 1.8), while high blood pressure was the factor associated with the highest risk of stroke (HR, 3.0; 95\% $\mathrm{CI}=2.0-4.4)$, also followed by metabolic syndrome (HR, 2.1; $95 \% \mathrm{CI}=1.5-2.9$ ).

The population attributable risk (PAR) fraction varied among those components. The low HDL cholesterol had the greatest PAR (39\%) to CHD events but the least PAR (10\%) to stroke. Instead, high blood pressure had the greatest impact (PAR, $45 \%)$ on stroke.
To estimate the discrimination ability of components and number of metabolic syndrome in the prediction of CHD and stroke, we calculated the area under the ROC curve. Using the numbers of metabolic syndrome as a single factor for both CHD and stroke prediction, the areas under the ROC curve were similar ( 0.76 for CHD and 0.78 for stroke). The measurements of discrimination ability showed that metabolic syndrome numbers had the highest $c$ statistics among all components of metabolic syndrome. Although high BMI and low HDL had the highest hazard risks for CHD and metabolic syndrome numbers had the highest discrimination ability, there were no statistically significant differences among the hazard risks and values of $c$ statistics. The calibrations of metabolic syndrome and components for predicting CHD and stroke events, partitioned by the Hosmer and Lemeshow test, were well fitted with the $P$ values of the chi-square test as 0.265 for CHD and 0.159 for stroke. In each gender, the metabolic syndrome numbers and components of metabolic syndrome still played important roles for coronary heart disease and

Table 3

Hazard risk (HR), 95\% confidence interval (CI) and estimated population attributable risk (PAR) by numbers of metabolic syndrome and component, controlling for sex, age smoking, drinking and physical activity status $(N=3515)$

\begin{tabular}{|c|c|c|c|c|c|c|c|c|c|c|c|}
\hline \multirow[t]{2}{*}{ Numbers } & \multirow[t]{2}{*}{$N$} & \multicolumn{5}{|c|}{ Coronary heart disease } & \multicolumn{5}{|l|}{ Stroke } \\
\hline & & $\begin{array}{l}\text { Hazard } \\
\text { risk }\end{array}$ & \multicolumn{2}{|c|}{$\begin{array}{l}95 \% \text { Confidence } \\
\text { interval }\end{array}$} & $P$ value & $\begin{array}{l}\text { Population } \\
\text { attributable } \\
\text { risk }\end{array}$ & Hazard risk & \multicolumn{2}{|c|}{$\begin{array}{l}95 \% \text { Confidence } \\
\text { interval }\end{array}$} & $P$ value & $\begin{array}{l}\text { Population } \\
\text { attributable } \\
\text { risk }\end{array}$ \\
\hline 0: Reference & 805 & 1 & & & & & 1 & & & & \\
\hline 1 & 1083 & 1.99 & 0.83 & 4.74 & 0.121 & 0.23 & 0.93 & 0.50 & 1.73 & 0.815 & 0.00 \\
\hline 2 & 779 & 4.00 & 1.74 & 0.0 & 0.001 & 0.40 & 2.20 & 1.25 & 3.89 & 0.007 & 0.21 \\
\hline 3 & 518 & 3.57 & 1.46 & 8.74 & 0.005 & 0.27 & 2.39 & 1.31 & 4.36 & 0.005 & 0.17 \\
\hline $4-5$ & 330 & 5.47 & 2.18 & 13.73 & 0.000 & 0.30 & 3.48 & 1.87 & 6.50 & $<.0001$ & 0.19 \\
\hline$P$ for trend & & $<0.0001$ & & & & & $<0.0001$ & & & & \\
\hline Metabolic syndrome & 848 & 1.83 & 1.16 & 2.90 & 0.010 & 0.17 & 2.05 & 1.45 & 2.91 & $<.0001$ & 0.20 \\
\hline High blood pressure & 1478 & 1.76 & 1.12 & 2.77 & 0.014 & 0.24 & 2.98 & 2.02 & 4.39 & $<.0001$ & 0.45 \\
\hline High body mass index & 517 & 2.22 & 1.32 & 3.75 & 0.003 & 0.15 & 1.69 & 1.09 & 2.60 & 0.019 & 0.09 \\
\hline High triglycerides & 869 & 1.89 & 1.20 & 2.97 & 0.006 & 0.18 & 1.62 & 1.14 & 2.31 & 0.007 & 0.13 \\
\hline Low HDL cholesterol & 1721 & 2.28 & 1.44 & 3.60 & 0.000 & 0.39 & 1.25 & 0.88 & 1.77 & 0.208 & 0.11 \\
\hline Hyperglycemia & 1115 & 1.17 & 0.75 & 1.83 & 0.478 & 0.05 & 1.67 & 1.20 & 2.34 & 0.003 & 0.18 \\
\hline
\end{tabular}


stroke events. The magnitudes of hazard risks, population attributable risk, and $c$ statistics were higher among men than women.

\section{Discussion}

We demonstrated that prevalence rates of metabolic syndrome increased progressively, especially among older women. Also, metabolic syndrome as an entity had the highest discrimination ability to specify cardiovascular events. There were several possible reasons to explain the increasing prevalence rates between two measurement periods. First, the study population got older and the atherosclerotic risk increased. Second, there might be changes in diet habits or physical activity over years. Metabolic syndrome was prevalent among US and European countries [20,21], and our study also showed high prevalence among ethnic Chinese. The data were compatible with other population-based studies [22]. Because the age distribution of various study samples, time to conduct the study and criteria were different, the comparison of metabolic syndrome prevalence only provided preliminary clues for further investigation. For ethnic Chinese, our study results were also compatible with adults in China $(9.8 \%$ in men and $17.8 \%$ in women by original NCEP-ATPIII criteria, from sampled subjects in the InterASIA study) [23], and Hong Kong Chinese (16.7\% in original NCEP-ATPIII, $21.2 \%$ in modified Asian ATP III) [24]. How to explain the rather high prevalence in this study population? First, the study subjects were rather elder population, with prone to high atherosclerotic risks. Second, different components and cutoff points made the comparison difficult. This study provided the repeated measures of metabolic syndrome components in longitudinal follow-up and we clearly showed the prevalence rates of metabolic syndrome increased progressively. Racial differences of metabolic syndrome prevalence were found. African Americans and American Indians had higher prevalence but their CHD risk was not proportionally increased [25]. First, the cohort was composed of an older population. Second, a relatively high prevalence of high blood pressure and low HDL cholesterol components among the cohort made the prevalence rates high. Further intervention on controlling blood pressure and raising HDL levels should be emphasized.

The estimated hazard risks of cardiovascular disease events associated with metabolic syndrome components were compatible with other community-based studies for the American-Indian [25], elderly [26], and Europeans [27,28]. Our data showed risks of metabolic syndrome did not have synergistic effects on CHD events. From Table 3, the estimated hazard risk of metabolic syndrome was similar to other risk component for CHD and stroke, while the estimated hazard of metabolic syndrome did not exceed the hazard of other risk component. The discrepancy can be explained by the losing power in binary status of metabolic syndrome in the model. By its components, there were sig- nificant dose-response relationships between the numbers of metabolic syndrome and cardiovascular events.

Our findings showed differences in men and women in terms of metabolic syndrome prevalence rates. Men still had higher incidence rates of CHD and stroke. Menopause might play an important factor for a higher prevalence of metabolic syndrome among women. The high prevalence of low HDL cholesterol and obesity also made the gender difference significant. Overall men had biological susceptibility for atherosclerosis, and the gender difference decreased among older populations [29].

Our study included waist circumference only in the second follow-up; instead, we used BMI as general obesity index to predict cardiovascular events in this study. It is possible to overestimate the prevalence of metabolic syndrome in baseline measurement by waist circumference criteria because waist circumference might fluctuate greater than BMI in the sequential measures. We used BMI component in baseline measurement. General obesity indicated by high BMI values, were strongly associated with risky cardiovascular biomarkers in the Women's Health Study population [30]. Also, BMI was proven as good criteria for screening Japanese population for metabolic syndrome [31], and the national survey data in Taiwan has demonstrated a significant relationship between BMI and waist circumference [32]. Among another Taiwanese cohort study, Chen et al. [33] demonstrated that central obesity could be replaced by body mass index for metabolic syndrome and the hazard risks for ischemic stroke by central obesity or body mass index were similar. Our study showed BMI-specific rates of metabolic syndrome were lower than waist circumference-specific metabolic syndrome rates.

Low HDL cholesterol and high blood pressure were the most prevalent and important components of metabolic syndrome in Taiwan. In contrast, central obesity is the highest risk attribute among American, and the rates of metabolic syndrome were higher for men than women [20]. It has been noted that cholesterol levels were lower in Asian populations [34] compared with that in Caucasians, implying that low HDL cholesterol has an important role in CHD events among Asians.

Other than individual disease prediction, metabolic syndrome as multiple risk clusters is a useful tool for identifying the magnitude of cardiovascular diseases in a community and measuring the abilities of discrimination and calibration. We found that subjects with metabolic syndrome had an increased likelihood of developing cardiovascular diseases. For Taiwanese, high co-morbidities, such as hypertension, hyperglycemia, and hyperlipidemia, are also demonstrated in other screening programs [35]. It is important to provide counseling on lifestyle modifications to persons with metabolic syndrome.

There were several limitations in this study. First, the incidence rate of CHD was rather low even after a decade of follow-up, thereby probably lessening the power to validate model fitting. Second, we did not stratify stroke events into 
subtypes. We integrated all types of stroke into one endpoint due to a large portion of unclassified stroke (around one third) and focused on the risk profiles on one stroke endpoint [9]. Also, we defined coronary percutaneous revascularization and bypass surgery as endpoints, procedures that were determined by physician behavior rather than biological consequence. It might not be good for epidemiologic studies on community.

The results implied that in low incidence of CHD and high stroke rates such as ethnic Chinese, metabolic syndrome played important roles. The clinical relevance and applications of the study are mandatory for lifestyle modification to prevent progress of metabolic syndrome. Further intervention programs should be implement to promote health life in the community.

In conclusion, our study suggests that metabolic syndrome is prevalent in the study community, especially among older women. Its components had risk effects on CHD and stroke events.

\section{Acknowledgments}

The authors wish to thank the cardiologists at the National Taiwan University Hospital for their invaluable assistance. This study was partly supported by the grants from the National Taiwan University (NTUH 2004S023) and National Science Council (NSC 2004-2314-B-002-218, 2003-2314B-002-197) in Taiwan.

\section{References}

[1] Reaven GM. Role of insulin resistance in human disease: Banting lecture 1998. Diabetes 1988;37:1595-607.

[2] DeFronzo RA, Ferrannini E. Insulin resistance. A multifaceted syndrome responsible for NIDDM, obesity, hypertension, dyslipidemia, and atherosclerotic cardiovasular disease. Diab Care 1991;14: 173-94.

[3] Boyko EJ, de Courten MM, Zimmet PZ, et al. Features of the metabolic syndrome predict higher risk of diabetes and impaired glucose tolerance: a prospective study in Mauritius. Diab Care 2000;23: 1242-8.

[4] Hanson RL, Imperatore G, Bennett PH, Knowler WC. Components of the "Metabolic Syndrome" and incidence of type 2 diabetes. Diabetes 2002;51:3120-7.

[5] Zimmet P, Alberti KG, Shaw J. Global and societal implications of the diabetes epidemic. Nature 2001;414:782-7.

[6] Executive Summary of The Third Report of The National Cholesterol Education Program (NCEP) Expert Panel on Detection, Evaluation, And Treatment of High Blood Cholesterol In Adults (Adult Treatment Panel III). JAMA. 2001; 285:2486-2497.

[7] WHO expert consultation. Appropriate body-mass index for Asian populations and its implications for policy and intervention strategies. Lancet 2004;363:157-63.

[8] Reddy KS. Cardiovascular disease in non-Western countries. N Engl J Med 2004;350:2438-40.

[9] Chien KL, Sung FC, Hsu HC, et al. Apolipoprotein A1 \& B, and stroke events in a community-based cohort in Taiwan: Report of Chin-Shan Community cardiovascular study. Stroke 2002;33:39-44.
[10] Lee YT, Lin RS, Sung FC, et al. Chin-Shan Community cardiovascular cohort in Taiwan: baseline data and five-year follow-up morbidity and mortality. J Clin Epidemiol 2000;53:836-46.

[11] Wong ND, Sciammarella MG, Polk D et al. The metabolic syndrome, diabetes, and subclinical atherosclerosis assessed by coronary calcium. J Am Coll Cardiol. 41:1547-53.

[12] Inoue S, Zimmet P. The Asia-Pacific perspective: redefining obesity and its treatment. Sidney: 2000, Health Communications Australia Pty Limited.

[13] The IDF consensus group: The IDF consensus worldwide definition of the metabolic syndrome http://www.idf.org/webdata/docs/ MetSyndrome_FINAL.pdf (access at 2006, April, 11).

[14] Grundy SM, Cleeman JI, Daniels SR, et al. Diagnosis and management of the metabolic syndrome: an American Heart Association/National Heart, Lung, and Blood Institute Scientific Statement. Circulation 2005;112:2735-52.

[15] Breslow NE, Day NE. Statistical methods in cancer research, vol. II. The design and analysis of cohort studies. Lyon: IARC; 1987.

[16] Grambsch PM, Therneau TM. Proportional hazards tests and diagnostics based on weighted residuals. Biometrika 1994;81:515-26.

[17] D'Agostino Sr RB, Grundy S, Sullivan LM, Wilson P. Validation of the Framingham coronary heart disease prediction scores: results of a multiple ethnic groups investigation. JAMA 2001;286: $180-7$.

[18] Hanley JA, McNeil BJ. The meaning and use of the area under a receiver operating characteristic (ROC) curve. Radiology 1982;143:29-36.

[19] Hosmer Jr DW, Lemeshow S. Applied logistic regression. New York: John Wiley \& Sons; 1989.

[20] Ford ES, Giles WH, Dietz WH. Prevalence of the metabolic syndrome among US adults: findings from the third National Health and Nutrition Examination Survey. JAMA 2002;287:356-9.

[21] Hu G, Qiao Q, Tuomilehto J, et al. Prevalence of the metabolic syndrome and its relation to all-cause and cardiovascular mortality in nondiabetic European men and women. Arch Intern Med 2004;164:1066-76.

[22] Eckel RH, Grundy SM, Zimmet PZ. The metabolic syndrome. Lancet 2005;365:1415-28.

[23] Gu D, Reynolds K, Wu X, et al. Prevalence of the metabolic syndrome and overweight among adults in China. Lancet 2005;365:1398405 .

[24] Thomas GN, Ho SY, Janus ED, et al. The US National Cholesterol Education Programme Adult Treatment Panel III (NCEP ATP III) prevalence of the metabolic syndrome in a Chinese population. Diab Res Clin Pract 2005;67:251-7.

[25] Resnick HE. Metabolic syndrome in American Indians. Diab Care 2002;25:1246-7.

[26] Lindblad U, Langer RD, Wingard DL, et al. Metabolic syndrome and ischemic heart disease in elderly men and women. Am J Epi 2001;53:481-9.

[27] Isomaa BM, Almgren PM, Tuomi TM, et al. Cardiovascular morbidity and mortality associated with the metabolic syndrome. Diab Care 2001;24:683-9.

[28] Lakka HMM, Laaksonen DE, Lakka TA, et al. The metabolic syndrome and total and cardiovascular disease mortality in middle-aged Men. JAMA 2002;288:2709-16.

[29] McGovern PG, Jacobs Jr DR, Shahar E, et al. Trends in acute coronary heart disease mortality, morbidity, and medical care from 1985 through 1997: the Minnesota heart survey. Circulation 2001;104: $19-24$.

[30] Mora S, Lee I, Buring JE, Ridker PM. Association of physical activity and body mass index with novel and traditional cardiovascular biomarkers in women. JAMA 2006;295:1412-9.

[31] Anuurad E, Shiwaku K, Nogi A, et al. The new BMI criteria for Asians by the regional office for the western Pacific region of WHO are suitable for screening of overweight to prevent metabolic syndrome in elder Japanese workers. J Occup Health 2003;45:335-43. 
[32] Pan WH, Flegal KM, Chang HY, et al. Body mass index and obesityrelated metabolic disorders in Taiwanese and US whites and blacks: implications for definitions of overweight and obesity for Asians. Am J Clin Nutr 2004;79:31-9.

[33] Chen HJ, Bai CH, Yeh WT, Chiu HC, Pan WH. Influence of metabolic syndrome and general obesity on the risk of ischemic stroke. Stroke 2006;37:1060-4.
[34] Ota TM, Takamura TM, Hirai NM, Kobayashi KM. Preobesity in World Health Organization classification involves the metabolic syndrome in Japanese. Diab Care 2002;25:1252-3.

[35] Chen TH, Chiu YH, Luh DL, et al. Community-based multiple screening model: design, implementation, and analysis of 42,387 participants. Cancer 2004;100:1734-43. 